\title{
A NEW METHOD FOR ESTIMATING THE NUMBER OF OBJECTS SATISFYING AN OBJECT-ORIENTED QUERY INVOLVING PARTIAL PARTICIPATION OF CLASSES
}

\author{
Wan-Sup Cho ${ }^{1}$, Chong-Mok Park ${ }^{1}$, Kyu-Young Whang ${ }^{1}$, and Sang-Hyuk Son ${ }^{2}$ \\ ${ }^{1}$ Department of Computer Science, Korea Advanced Institute of Science and Technology, Taejon, 305-701, Korea \\ ${ }^{2}$ Department of Computer Science, University of Virginia, Charlottesville, VA 22903, USA
}

(Received 8 February 1995; in final revised form 15 September 1995)

\begin{abstract}
The intermediate result cardinality - the number of objects satisfying a condition given in a query - is an important factor for estimating the cost of the query in query optimization. In this paper we show that an object-oriented query often involves partial participation of classes in a relationship. We then present a new technique for estimating the intermediate result cardinality in such a query. Partial participation has not been considered seriously in existing techniques. Since the proposed technique uses detailed statistics to accommodate partial participation, it estimates the intermediate result cardinality more accurately than existing ones. We also show that these statistics are easily obtained by using inherent properties of object-oriented databases.
\end{abstract}

Key words: object-oriented databases, query optimization, cost model, selectivities, intermediate results

\section{INTRODUCTION}

Object-oriented database management systems (OODBMSs) are adequate for supporting new database applications such as engineering databases and multimedia databases $[1,12,17]$. Although there are a number of OODBMSs, most of the current OODBMSs do not provide sufficient support for the database management facilities [13, 17], such as high-level query languages, query optimization, dynamic schema changes, indexing techniques, concurrency control, and recovery. Among these facilities, query optimization is essential for efficient processing of high-level queries.

In query optimization, the system automatically generates a set of reasonable execution plans for processing a given query, and selects the one with the minimum estimated cost. One of the most important factors that affects the cost estimation of an execution plan is the intermediate result cardinality - the number of objects in an intermediate result $[3,7,19,21]$. This is because the intermediate result becomes the operand of the next operation in the execution plan, and the cost of the next operation can be estimated mainly based on the number of objects in its operands [18, 19, 21]. Hence, accurate estimation of intermediate result cardinality is a prerequisite for good query optimization.

Intermediate results cardinalities are estimated by multiplying the cardinalities of unconditional joins $^{\dagger}[24]$ with the selectivity factors of conditions [19, 21]. The selectivity factor of a condition represents the portion of objects that satisfy the condition. In this paper, we propose new techniques for estimating the cardinalities of unconditional joins, selectivity factors, and intermediate results cardinalities in object-oriented queries.

We first show that an object-oriented query often involves partial participation of classes in a relationship and compare the case with that for relational queries. Partial participation means that only a proper subset of objects in a class are related to the objects of another class [6]. Two objects are related (or has a relationship) if an attribute in one object has the Oid of another object as its value. For example, if only a subset of objects in the class Student are related to the objects in the class Car via the relationship own, then the class Student partially participates in the relationship own. Here, we call the class Student a partial participation class with respect to the relationship own.

\footnotetext{
${ }^{\dagger} \mathrm{A}$ join without selection conditions on either relation.
} 
We then propose new techniques for estimating the cardinalities of unconditional joins and selectivity factors of a query involving partial participation classes. Partial participation has not been considered seriously in the literature [15]. Most of existing query optimization techniques [2, $8,11,16,19]$ except for Whang et al.[24, 21, 22] have not considered partial participation. We discuss in Section 4.1 that these conventional techniques often incur large estimation errors for the queries involving partial participation classes. We also consider the effect of multi-valued attributes on the estimation of intermediate results cardinalities. Most of existing estimation techniques [2, $16,19,24,21]$ have not considered multi-valued attributes seriously. The proposed technique for estimating the cardinalities of unconditional joins extends Whang's technique [24, 21, 22] so as to consider the characteristics of object-oriented databases. The proposed techniques require new types of statistics for estimating query costs. We also show that these statistics can be easily obtained by taking advantage of inherent properties of object-oriented databases.

The paper is organized as follows. In Section 2, we briefly review the basic concepts of objectoriented databases necessary for discussing query optimization. In Section 3, we explain the reasons why partial participation occurs frequently in object-oriented queries and compare the case with that for relational queries. In Section 4, we discuss the impact of partial participation and multi-valued attributes on the estimation of the intermediate results cardinalities and present new estimation techniques. We then compare our techniques with the conventional ones to show the significance of the role of partial participation. Finally, we conclude the paper in Section 5.

\section{BACKGROUND}

In this paper, we use a generally accepted subset of object-oriented data models described in the literature [1, 12]. Objects are uniquely identified by object identifiers (Oids). Objects having the same properties (attributes and methods) are classified into a class. The definition of a class forms a two-dimensional rooted directed graph of classes called the schema graph for that class [12].

Fig. 1 is an example schema graph for the class Person. The class Person is the root of the aggregation graph that includes the classes Vehicle and Company. The class Person is also the root of the inheritance graph (or class hierarchy) involving the classes Employee and Student. For any



Fig. 1: Schema Graph for the Person, Vehicle, and Company classes.

class $C, C^{*}$ denotes all the classes in the inheritance graph rooted at the class $C$. For example, Person* denotes the (union of the) classes Person, Employee, and Student, which belong to the inheritance graph rooted at the class Person. All the properties of a superclass are inherited to its subclasses.

An attribute whose domain is a class in a schema graph represents a relationship between the class in which the attribute is defined and the class that is the domain of the attribute. For example, the attribute own represents the relationship between the classes Person and Vehicle. The domain of an attribute may be the union of a given class and all its subclasses [12]. For example, in Fig. 1, the domain of the attribute own is the entire inheritance graph rooted at Vehicle, i.e., Vehicle*, and the values of the attribute own are the Oids referring to the objects of the class Vehicle or any subclass of it. If a class is the domain of more than one attribute, we call the class a common domain for the attributes. For example, in Fig. 1, the class Company is the common domain of the two attributes manuf and workfor. If an attribute has a single value for a particular object, we call it a single-valued attribute; otherwise, i.e., if it may have more than one value, we call it a multi-valued attribute. 
Most of the object-oriented query languages [10,12] are extensions of relational ones supporting object-oriented concepts $[1,12]$. In this paper, we use the query model proposed by Kifer et al.[10]. Concentrating on the nested predicates in which methods and views are excluded. Although this kind of query is a small part of Kifer's query model, it is an important class of queries that needs careful investigation on the costs for effective query optimization because nested predicates involve multiple expensive joins.

Example 1 In Fig. 1 consider the following query for finding the names of all persons who own a truck manufactured by "Ford". Here we assume that the attribute own is a single-valued attribute.

$\begin{array}{ll}\text { SELECT } & \text { p.name } \\ \text { FROM } & \text { Person p } \\ \text { WHERE } & \text { p.own[Truck].manuf.name = "Ford"; }\end{array}$

An important feature of Kifer's query model is the extended path expression (or simply the path ) [10] which has the following form

$$
P=C_{0} \cdot A_{1}\left\{\left[C_{1}\right]\right\} \cdot A_{2}\left\{\left[C_{2}\right]\right\} \ldots A_{n}\left\{\left[C_{n}\right]\right\}, \quad n \geq 0
$$

where $n \geq 0, C_{0}, \ldots, C_{n}$ are class names, $A_{1}, \ldots, A_{n}$ are attribute names, and braces denote optional terms (i.e., only $C_{0}$ is mandatory), If "[ $\left.C_{i}\right]$ " is omitted, we assume that the domain of $A_{i}$ is the class (or inheritance graph) that is defined in the schema as the domain of $A_{i}$. The length of the path $P$ is defined as the number of attributes, $n$, in $P$. If the length of a path is zero, it represents just a class. We call the first class $C_{0}$ the starting class and the last attribute $A_{n}$ the ending attribute of the path $P[2]$.

In the path $P$, we call $C_{i-1}$ the codomain and $C_{i}$ the domain of the attribute $A_{i}$. Note that users can limit the domain of $A_{i}$ to a specific class (or its inheritance graph) by designating the class in the brace followed by $A_{i}$. We call this designation domain substitution of $A_{i}$. If the domain of $A_{i}$ is substituted by the class $C_{i}$, the codomain of $A_{i+1}$ is automatically limited to (or substituted by) $C_{i}$. We call this limitation codomain substitution of $A_{i+1}$. As an exception, codomain substitution for the first attribute $A_{1}$ occurs when the starting class is designated to be a specific class rather than the union of all the classes in an inheritance graph. For example, in the path Student.own[Truck].color, the codomain for the attribute own is limited to the class Student, and the domain to the class Truck, i.e., we have both codomain and domain substitutions.

The path is a convenient and natural way of expressing queries along the aggregation graph [10, 12]. Furthermore, domain and codomain substitutions in a path allow users to express more delicate queries $[10,12]$.

Example 2 In Fig. 1, consider a path Person*.own.manuf.name. We can substitute the codomain and domain classes of the attribute own in the path in various ways as follows. For simplicity, we abbreviate the class names Person, Employee, Student, Vehicle, Truck, and Car as P, E, S, V, T, and C.

$$
\begin{array}{lll}
P_{1}: \text { P.own[}\left[\mathrm{V}^{*}\right] \text {.manuf.name } & P_{5}: \text { E.own[}\left[\mathrm{V}^{*}\right] \text {.manuf.name } & P_{9}: \text { S.own[}\left[\mathrm{V}^{*}\right] \text {.manuf.name } \\
P_{2}: \text { P.own[V].manuf.name } & P_{6}: \text { E.own[V].manuf.name } & P_{10}: \text { S.own[V].manuf.name } \\
P_{3}: \text { P.own[C].manuf.name } & P_{7}: \text { E.own[C].manuf.name } & P_{11}: \text { S.own[C].manuf.name } \\
P_{4}: \text { P.own[T].manuf.name } & P_{8}: \text { E.own[T].manuf.name } & P_{12}: \text { S.own[T].manuf.name }
\end{array}
$$

The path $P_{1}$ denotes the manufacturer's names of vehicles (including trucks and cars) owned by persons. Similarly, path $P_{12}$ denotes the manufacturer's names of trucks owned by students. The rest can be explained similarly.

In a path, each attribute whose domain is a class implicitly specifies a join between codomain and domain classes of the attribute. We call this kind of join implicit join [8, 10, 12, 16]. For example, two implicit joins are specified in the path Person.own[Vehicle].manuf.name: (1) one between Person and Vehicle via the attribute own, and (2) another between Vehicle and Company via the attribute manuf. All joins appearing in the remainder of this paper are implicit joins. 
In Eq.(1), we call a sequence of $n+1$ objects $\left[o_{0}, o_{1}, \ldots, o_{n}\right]$ a path instance of the path $P$ if it satisfies all of the following conditions $[2,10]:(1) o_{0}$ is an object (or instance) of the class $C_{0}$, and (2) $o_{i}(0<i \leq n)$ is the value of the attribute $A_{i-1}$ of object $o_{i-1}$. For example, the sequence of objects [p, t, c, “Jones"] is a path instance for Person.own[Truck].manuf.name, where $\mathrm{p}$ is a Person object, p.own has the Oid of the Truck object $t$, t.manuf has the Oid of the Company object $c$, and c.name is the string "Jones".

We use the following statistics reflecting the state of the database for the path $P$. We present an efficient method for obtaining these statistics in Sections 4.2 and 4.3.

- $n(P)$ : the number of path instances in the path $P$. If the length of the path $P$ is zero, $n(P)$ is the number of objects of the class specified by $P$. For example, $\mathrm{n}$ (Student) is just the number of Student objects. On the other hand, $n$ (Student.own[Car]) is the number of pairs of objects (i.e., path instances) [s, c], where s is an object in Student, c in Car, and s.own contains the Oid of $c$.

- $d v(P)$ : the number of distinct values of the ending attribute of the path $P$ in the path instances. For example, dv(Student.name) is the number of distinct name in the path instances for Student.name, and dv(Student.own[Truck].manuf) is the number of distinct Company objects in the path instances for Student.own[Truck].manuf.

We make the following assumptions.

- For considering codomain/domain substitution in an object-oriented query, we extend the definition of the uniform distribution $[4,19]$ of an attribute as follows: If the domain of a complex attribute $A_{i}$ consists of $D_{1}, \ldots, D_{n}, n>1$, then the Oids of individual $D_{k}$ 's that appear in $A_{i}$ of $C_{i}$ are uniformly distributed in the class $C_{i}, i=1,2, \ldots$ For example, in Fig. 1, the Oids of Car that appear in the attribute own are uniformly distributed in each of Person, Employee, and Student classes. Similarly, the Oids of Vehicle and Truck are also uniformly distributed in each of these classes. Note that this assumption does not modify the commonly accepted definition of uniform distribution [4, 19], but extends the definition according to the subclasses in the class hierarchy. If $n=1$, i.e., the inheritance graph consists of a single class, then this assumption is reduced to the conventional definition $[4,19]$.

- An Oid consists of a <class-id, instance-id> pair, where class-id is the identifier of the class to which the object belongs, and instance-id is the identifier of an object (instance) either within the class or within the entire database[12]. This kind of Oid structure is used in ORION [12].

\section{PARTIAL PARTICIPATION OF CLASSES}

In this section we show that an object-oriented query often involves partial participation of classes in a relationship and compare the case with that for relational queries. We also discuss the effect of partial participation on the estimation of the cardinalities for unconditional joins.

A relational join is typically done between a foreign key attribute (FKA) and a primary key attribute (PKA); here, both relations of the join may be partial participation relations. We call such a join a partial participation join. If the FKA allows null values, then the tuples having null values for the FKA do not participate in the join. Thus, the relations including the FKA partially participates in the join. Likewise, if only a subset of values of the PKA is referred to by the values of the FKA, then the relation including the PKA partially participates in the join. Tuples that do not participate in the join are called dangling tuples [6, 15]. For example, consider a join between Car and Person where Car.owner is a foreign key corresponding to the primary key Person.id. Then, the Car tuples that have null values in Car.owner (i.e., cars with no owner) are dangling tuples. Likewise, the Person tuples that are not referred to by Car.owner (i.e., persons having no cars) are also dangling tuples. Much of the work on relational query optimization ignores the effect of partial participation joins [15, 18, 19]. This is because it is assumed that referential integrity generally holds and the tuples of the FKA-side relation totally participate in the join. However, 
as indicated by Korth and Silberschatz[15], if both relations have dangling tuples, the selectivity factor produced by Selinger's method [19] is higher than the correct value. As a correction factor for partial participation joins, Whang et al. [24, 21, 22] introduced the concept of join participation ratio (defined originally in [24] as join selectivity). We explain the concept in Section 4.1 in detail.

Another source of partial participation joins in relational databases is the appropriate use of selection and join operations. After selection operations are performed, a join becomes a partial participation join because only the qualified objects participate in the join. We call this kind of partial participation partial participation by selections.

There is an important difference between partial participation by selections in relational databases and the partial participation in object-oriented databases. In partial participation by selections, since the proportion of participating objects to the entire objects depends on the selectivity factor of the selection operations, it is difficult to reflect the effect of partial participation in query optimization. We need to handle this effect via either assuming attribute independence [18, 19] or maintaining a special data structure for keeping the joint distribution of selection and join attributes [18]. However, it has been argued that attribute independence leads to the upper bound of the actual expected cost [4] and maintaining special data structures may be too expensive[18]. On the other hand, partial participation in object-oriented databases is visible through existence of subclasses, which corresponds to the results of selection operations in relational databases, and domain/codomain substitutions in queries. Since subclasses are modelled at the schema level, we can easily obtain the statistics needed to account for partial participation from these subclasses and maintain them in the system catalog. The detailed method for obtaining these statistics is fully discussed in Section 4.2 and Section 4.3.

In object-oriented queries, partial participation joins occur frequently. These are mainly caused by codomain and/or domain substitutions and common domains. We use Fig. 2 as an example. It consists of two class hierarchies rooted at the classes $C$ and $D$. The attribute $A_{i}$ is defined for the class $C$ and is inherited to the subclasses. The domain of $A_{i}$ is the entire class hierarchy rooted at the class $D$ (i.e., $D^{*}$ ). The grey-colored subregions of $C_{i}$ and $D_{j}$ denote the objects participating in the join $C_{i} \cdot A_{i}\left[D_{j}\right]$, and the rest denote those not participating in the join. We identify three cases where partial participation occurs by using the schema graph in Fig. 2.

The first case occurs when the domain of an attribute is substituted in a path expression. In this case, only a subset of objects in the codomain class of the attribute refer to the objects in the substituted domain. For example, suppose the domain of $A_{i}$ is substituted by $D_{j}$ in Fig. 2. Then, since $C^{*}$ objects refer to the $D^{*}$ objects, only a subset of $C^{*}$ objects may refer to $D_{j}$ objects. Thus, the codomain of $A_{i}$ (e.g., $C_{i}$ ) partially participate in the join with $D_{j}$.

The second case occurs when the codomain of an attribute is substituted in a path expression. Then, only a subset of objects in the domain class of the attribute are referred to by the objects in the substituted codomain. For example, suppose the codomain of $A_{i}$ is substituted by $C_{i}$, which is a subclass of $C$. Then, since $D^{*}$ objects are referred to by $C^{*}$ objects, only a subset of $D^{*}$ objects are likely to be referred to by $C_{i}$ objects. Thus, the domain of $A_{i}$ (e.g., $D_{j}$ ) partially participates in the join with $C_{i}$. We justify this case more formally as follows. The distribution of the values of the attribute $A_{i}$ across the classes in the class hierarchy rooted at $C$ can be classified into three cases [14]. Here, we assume that the domain of the attribute $A_{i}$ is $D^{*}$.

- disjoint: each $D^{*}$ object is referred to by one class of $C^{*}$.

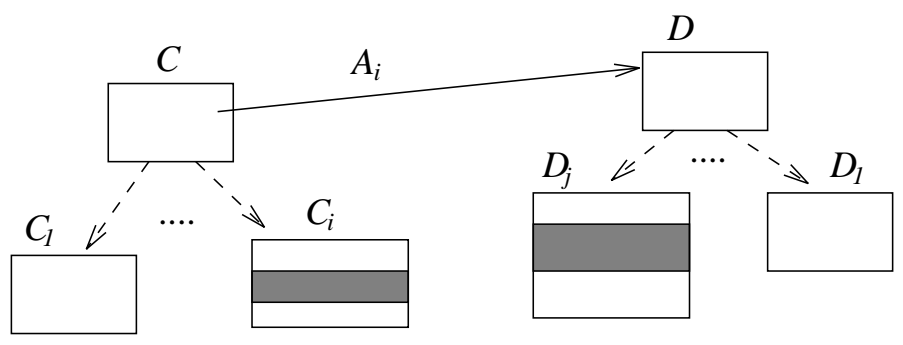

Fig. 2: A schema graph. 
- total inclusive: each $D^{*}$ object is referred to by any class in $C^{*}$.

- partial inclusive: the cases neither disjoint nor total inclusive.

Except for the total inclusive case, the second reason is satisfied because some objects of $D^{*}$ are not referred to via $C_{i} . A_{i}$.

The third case occurs when a class in a path is the common domain of multiple attributes, each of which refers to only a subset of the objects in the domain class. For example, suppose that the class $D_{j}$ in $C_{i} . A_{i}\left[D_{j}\right]$ is also the domain of the attribute $C^{\prime} . A_{i}^{\prime}$. Then, $D_{j}$ is the common domain of the attributes $C_{i} . A_{i}$ and $C^{\prime} . A_{i}^{\prime}$. Here, since only a subset of $D_{j}$ objects may be referred to by $C_{i} . A_{i}$ (the remainder of $D_{j}$ objects being referred to by $C^{\prime} . A_{i}^{\prime}$ ), the domain of $A_{i}$ (i.e., $D_{j}$ ) partially participates in the join with $C_{i}$.

For these reasons, object-oriented queries involve partial participation more frequently than relational ones. Let us note that, in Example 2, all the paths except for $P_{1}, P_{5}$, and $P_{9}$ include both codomain and domain substitutions, and thus involve partial participation join. Furthermore, in the path $P_{12}$ : S.own[T].manuf.name, if few students have a small portion of trucks, as is likely to happen in a real world, then most of the objects in the Student and Truck become dangling objects. Thus, the effects of partial participation in object-oriented databases are more significant than in relational databases.

\section{ESTIMATION OF INTERMEDIATE RESULTS CARDINALITIES}

In this section, we clarify the effects caused by partial participation in detail and propose new techniques for estimating the cardinalities of unconditional joins, selectivity factors, and intermediate results cardinalities that take the effects into account. In Section 4.1, we discuss the problems of conventional techniques for estimating the cardinalities of unconditional joins when they are used for partial participation joins. In Section 4.2, we present a new estimation technique that takes the effect of partial participation and multi-valued attributes into account. In Section 4.3, we discuss the effect of partial participation on the estimation of selectivity factors for conditions and present a new selectivity estimation technique. In Section 4.4, we show how to estimate intermediate results cardinalities for the queries involving $n$-way joins with selection conditions by using the cardinalities of unconditional joins and the selectivity factors. We then compare our estimation techniques with other existing ones.

\subsection{Problems of Conventional Techniques for Estimating the Cardinalities of Joins}

Many techniques for estimating the cardinality of a join have been proposed in the literature for relational databases [18, 19, 21]. We use the relational join $C_{i} \bowtie_{A_{i}=A_{j}} D_{j}$ as an example. We assume that no selection conditions are applied to individual relations (classes) until Section 4.3.

Selinger et al. [19] assume (1) uniform distribution [4], (2) attribute independence [4], and (3) referential integrity; that is, each value in the join attribute with the smaller cardinality has a matching value in the other join attribute. Under these assumptions, they estimate the cardinality of the join as follows:

$$
n\left(C_{i} \bowtie D_{j}\right)=n\left(C_{i}\right) \times n\left(D_{j}\right) \times \frac{1}{\max \left[d v\left(C_{i} \cdot A_{i}\right), d v\left(D_{j} \cdot A_{j}\right)\right]}
$$

Here, $n\left(C_{i}\right) \times n\left(D_{j}\right)$ is the cardinality of the Cartesian product between $C_{i}$ and $D_{j}$, and $1 / \max [\operatorname{dv}($ $\left.\left.C_{i} . A_{i}\right), d v\left(D_{j} . A_{j}\right)\right]$, the selectivity factor for the join, is the probability that a pair of tuples randomly selected from the Cartesian product satisfies the join condition. However, if the join is a partial participation join, the assumption (3) no longer holds. In Eq.(2), since $n\left(C_{i}\right) \geq$ $d v\left(C_{i} . A_{i}\right)$ and $n\left(D_{j}\right) \geq d v\left(D_{j} . A_{j}\right)$, the estimated cardinality is always greater than or equal to $\min \left[n\left(C_{i}\right), n\left(D_{j}\right)\right]$. Thus, the larger the portions of the dangling tuples in $C_{i}$ and $D_{j}$ become, the more error the estimation of the cardinality would produce. In an extreme, when only one pair of tuples from $C_{i}$ and $D_{j}$ participates in the join, the estimated cardinality is at least $\min \left[n\left(C_{i}\right), n\left(D_{j}\right)\right]$ times larger than the actual count. Thus, a correction factor should be applied 
to the estimates. We note that when assumption (3) as well as assumptions (1) and (2) holds, Eq. (2) gives the correct result.

Whang et al. [24, 21, 22] consider the effect of partial participation in estimating the cardinality of the join. They define the join participation ratio $J\left(C_{i}\right)$ (defined originally as the join selectivity [24]) for the relation $C_{i}$ as the ratio of the number of distinct join column values of the tuples participating in the unconditional join to the total number of the distinct join column values of $C_{i}$. They also assume that the relationship between $C_{i}$ and $D_{j}$ is many-to-one. ${ }^{\dagger}$ Under this assumption, they estimate the cardinality for $C_{i} \bowtie_{A_{i}=A_{j}} D_{j}$ as follows:

$$
n\left(C_{i} \bowtie D_{j}\right)=n\left(C_{i}\right) \times J\left(C_{i}\right) \times \frac{n\left(D_{j}\right)}{d v\left(D_{j} \cdot A_{j}\right)}
$$

Here, $n\left(C_{i}\right) \times J\left(C_{i}\right)$ is the number of tuples in $C_{i}$ that participate in the join, and $\frac{n\left(D_{j}\right)}{d v\left(D_{j} \cdot A_{j}\right)}$ is the average number of tuples in $D_{j}$ that have the same value in the attribute $A_{j}$. In Eq. (3), since the join selectivity $J\left(C_{i}\right)$ decreases as the portion of dangling tuples in $C_{i}$ increases, the effect from partial participation join is accounted for.

Since the estimation techniques proposed by Selinger et al. [19] and Whang et al. [24, 21, 22] are for the relational model, they have not considered the characteristics of object-oriented databases, such as multi-valued attributes, domain and codomain substitutions. Thus, these techniques are not appropriate for estimating the cardinalities of object-oriented queries.

In object-oriented databases, most literature assumes a simple query model in which no null values and no domain substitutions are allowed. Partial participation does not occur frequently in such a query model. Most of the research on query optimization and physical database design [2, $8,11,16]$ uses the average fan-out fan and the sharing level share as a part of the cost model. Let us consider a path expression $C_{i} . A_{i}\left[D^{*}\right]$ on the schema graph shown in Fig. 2. The fan-out, $\operatorname{fan}\left(C_{i} \cdot A_{i}\right)$, is the average number of $D^{*}$ objects referred to by an object of $C_{i}$. Similarly, the sharing level, share $\left(C_{i} . A_{i}\right)$, is the average number of $C_{i}$ objects that refer to the same object of $D^{*}$. By using these parameters, the cardinality of the join $C_{i} \cdot A_{i}\left[D^{*}\right]$ can be estimated in two different ways depending on the directions of the traversal ${ }^{\dagger}$ as follows $[2,11]$ :

$$
\begin{aligned}
& n\left(C_{i} \bowtie D^{*}\right) \quad=n\left(C_{i}\right) \times \operatorname{fan}\left(C_{i} . A_{i}\right) \quad /^{*} \text { forward traversal */ } \\
& n\left(C_{i} \bowtie D^{*}\right)=n\left(D^{*}\right) \times \operatorname{share}\left(C_{i} . A_{i}\right) \quad /^{*} \text { backward traversal */ }
\end{aligned}
$$

Bertino et al. [2] assume only single-valued attributes exist without null values and codomain and/or domain substitutions. Under these assumptions, $\operatorname{fan}\left(C_{i} . A_{i}\right)$ is estimated as 1 , and $\operatorname{share}\left(C_{i} . A_{i}\right)$ as $\frac{n\left(C_{i}\right)}{d v\left(C_{i} \cdot A_{i}\right)}$. Kim et al. [11] assume no null values and no domain substitutions exist. Under these assumptions, $\operatorname{fan}\left(C_{i} . A_{i}\right)$ is estimated as 1 if $A_{i}$ is a single-valued attribute; otherwise it is estimated as $\frac{n\left(C_{i} \cdot A_{i}\right)}{n\left(C_{i}\right)}$. However, Kim et al. [11] have not proposed the sharing level. Kemper [8] and Lanzelotte [16] consider null values and multi-valued attributes with no codomain and/or domain substitutions. They estimate $\operatorname{fan}\left(C_{i} . A_{i}\right)$ as 1 and $\operatorname{share}\left(C_{i} . A_{i}\right)$ as $\frac{n\left(C_{i}\right)}{d v\left(C_{i} . A_{i}\right)}$ when $A_{i}$ is a singlevalued attribute. However, they assume that fan-outs and sharing levels for multi-valued attributes are supplied by users.

These conventional estimation techniques have the following problems if they are used for object-oriented queries involving partial participation joins or multi-valued attributes. First, when $C_{i}$ is a partial participation class (i.e., $C_{i} . A_{i}$ has null values or the domain of $C_{i} . A_{i}$ is substituted), $\operatorname{fan}\left(C_{i} . A_{i}\right)$ is likely to be less than 1 even though the attribute $A_{i}$ is a single-valued attribute. For example, if only half of the $C_{i}$ objects participate in the join, $\operatorname{fan}\left(C_{i} . A_{i}\right)$ would be 0.5 instead of 1 . Second, the parameter $\frac{n\left(C_{i} \cdot A_{i}\right)}{n\left(C_{i}\right)}$ in $\operatorname{Kim}[11]$ has a fixed value independent of domain substitution of $A_{i}$, thus, cannot reflect the effect of domain substitution. A similar argument holds for the parameter $\frac{n\left(C_{i}\right)}{d v\left(C_{i} \cdot A_{i}\right)}$ in Bertino [2], Lanzelotte[16], and Kemper [8]. Third, since most of the

\footnotetext{
${ }^{\dagger}$ In the relational database design, a many-to-many relationship between entity sets is transformed into two one-to-many relationships between relations [6].

$\dagger$ There are two possible ways to traverse the classes in a path [11]: the forward traversal visits the classes from the codomain of an attribute to its domain, and the reverse traversal in the opposite direction.
} 
conventional estimation techniques assume single-valued attributes, it is natural to expect errors in estimation when multi-valued attributes are involved in the query. For example, if $A_{i}$ is a multivalued attribute and has $d$ values on the average, then $\operatorname{share}\left(C_{i} . A_{i}\right)$ in Bertino [2], Lanzelotte [16], and Kemper [8], should be multiplied by $d$.

The following example shows the error in estimation when using conventional methods for object-oriented queries involving partial participation joins. (Selinger's and Whang's methods are not compared since they are developed for the relational model.)

Example 3 Consider the path Student.own[Truck] in Fig. 1. For convenience, we abbreviate the class names Student and Truck as S and R. We assume that the attribute own is single-valued and does not allow null values to conform to the assumptions of the conventional techniques $[2,8,11,16]$. We also assume the following statistics:

$$
n(S)=1,000 ; n(S . o w n)=1,000 ; d v(S . o w n)=500 ; n(S . o w n[R])=50 ; n(R)=2,000 .
$$

Note that the join Student.own[Truck] is a partial participation join in which only 50 students (among 1,000 students) have less than 50 trucks (among 2,000 trucks) since $\mathrm{n}(\mathrm{S}$.own[R]) $=50$, the attribute own is single-valued, and some trucks may be shared. Conventional techniques $[2,8,16]$ estimate $\operatorname{fan}($ S.own $)$ as 1 and $\operatorname{share}($ S.own $)$ as $\frac{n(S)}{d v(S . \text { own })}=\frac{1,000}{500}$ regardless of the actual number of trucks referenced by the attribute own. These estimates are correct only when all students refer only to trucks. The cardinality of the join using these parameters can be estimated as follows depending on the join directions $[2,11]$ :

$$
\begin{array}{lll}
n(S \bowtie R) & =n(S) \times \operatorname{fan}(\operatorname{S.own})=1,000 \times 1=1,000 & / * \text { forward traversal */ } \\
n(S \bowtie R) & =n(R) \times \operatorname{share}(\text { S.own })=2,000 \times \frac{1,000}{500}=4,000 & / * \text { backward traversal */ }
\end{array}
$$

However, since each of the 50 students have a truck, the correct value of $n(S \bowtie R)$ is 50 .

\subsection{Estimation of Cardinalities of Joins}

In this section, we present a new technique for estimating the cardinalities of joins in objectoriented queries. This technique takes into account the effects of partial participation and multivalued attributes.

Let us consider the join $C_{i} \cdot A_{i}\left[D_{j}\right]$ shown in Fig. 2 again. For each codomain and domain pair $\left(C_{i}, D_{j}\right)$ of the attribute $A_{i}$, we define the fan-out as the average number of $D_{j}$ objects referred to by an object of $C_{i}$ and denote it as $\operatorname{fan}\left(C_{i} . A_{i}\left[D_{j}\right]\right)$. Similarly, we define the sharing level as the average number of $C_{i}$ objects that refer to the same object of $D_{j}$ and denote it as $\operatorname{share}\left(C_{i} \cdot A_{i}\left[D_{j}\right]\right)$. We calculate these parameters as follows:

$$
\begin{aligned}
\operatorname{fan}\left(C_{i} \cdot A_{i}\left[D_{j}\right]\right) & =\frac{n\left(C_{i} \cdot A_{i}\left[D_{j}\right]\right)}{n\left(C_{i}\right)} \\
\operatorname{share}\left(C_{i} \cdot A_{i}\left[D_{j}\right]\right) & =\frac{n\left(C_{i} \cdot A_{i}\left[D_{j}\right]\right)}{n\left(D_{j}\right)}
\end{aligned}
$$

Here, since $n\left(C_{i} . A_{i}\left[D_{j}\right]\right)$ is the total number of Oids (i.e., references) including duplicates in $C_{i} . A_{i}$ that refer to $D_{j}$ objects, each $C_{i}$ object has $\frac{n\left(C_{i} \cdot A_{i}\left[D_{j}\right]\right)}{n\left(C_{i}\right)}$ Oids of $D_{j}$ objects on the average. Similarly, we calculate share $\left(C_{i} \cdot A_{i}\left[D_{j}\right]\right)$ as in Eq. (7). Fig. 3 shows $\operatorname{fan}\left(C_{i} \cdot A_{i}\left[D_{j}\right]\right)$ and $\operatorname{share}\left(C_{i} \cdot A_{i}\left[D_{j}\right]\right)$ graphically.

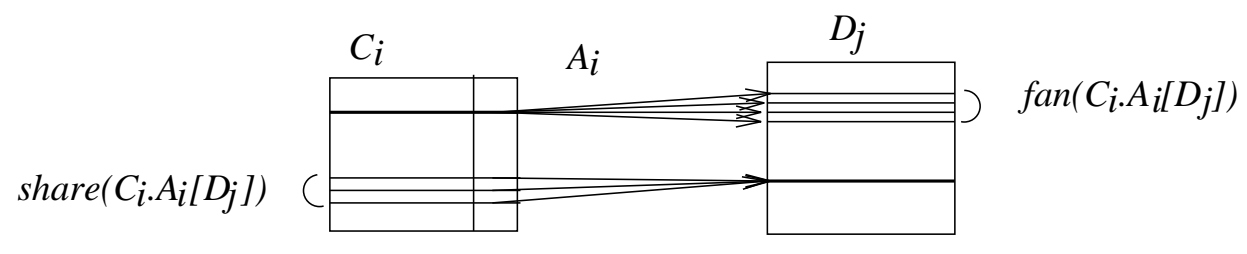

Fig. 3: $\operatorname{fan}\left(C_{i} \cdot A_{i}\left[D_{j}\right]\right)$ and $\operatorname{share}\left(C_{i} \cdot A_{i}\left[D_{j}\right]\right)$ 
The fan and share of Eqs. (6) and (7) consider partial participation and multi-valued attributes. For single-valued attributes, as the numbers of dangling objects in $C_{i}$ and $D_{j}$ increase, the fan and share defined in Eqs. (6) and (7) decrease proportionally. Specifically, if the attribute $A_{i}$ has null values or the domain of $A_{i}$ is substituted (i.e., $C_{i}$ partially participates in the join), then $n\left(C_{i} \cdot A_{i}\left[D_{j}\right]\right)$ may become less than $n\left(C_{i}\right)$. Hence, $\operatorname{fan}\left(C_{i} \cdot A_{i}\left[D_{j}\right]\right)$ may also become less than 1. Similarly, if the codomain of $A_{i}$ is substituted or if the class $D_{j}$ is a common domain (i.e., $D_{j}$ partially participates in the join), then $\operatorname{share}\left(C_{i} \cdot A_{i}\left[D_{j}\right]\right)$ may also become less than $\frac{n\left(C_{i}\right)}{d v\left(C_{i} \cdot A_{i}\right)}$. For multi-valued attributes with $d$ values on the average, the fan-outs and the sharing levels may be as much as $d$ times greater than those of single-valued attributes. These results contrast with those of the previous methods, in which the fan-out and the sharing level are fixed as 1 and $\frac{n\left(C_{i}\right)}{d v\left(C_{i} \cdot A_{i}\right)}$ $(\geq 1)$ regardless of the presence of dangling objects.

By using the fan-out and the sharing level defined in Eqs. (6) and (7), we estimate the cardinality of the join $C_{i} \cdot A_{i}\left[D_{j}\right]$ as follows. We use two formulas depending on the join directions:

$$
\begin{array}{lll}
n\left(C_{i} \bowtie D_{j}\right) & =n\left(C_{i}\right) \times \operatorname{fan}\left(C_{i} \cdot A_{i}\left[D_{j}\right]\right) & / * \text { forward traversal */ } \\
n\left(C_{i} \bowtie D_{j}\right) & =n\left(D_{j}\right) \times \operatorname{share}\left(C_{i} \cdot A_{i}\left[D_{j}\right]\right) & / * \text { backward traversal */ }
\end{array}
$$

Example 4 We estimate the cardinality of the join in Example 3 using the proposed method. Note that these results match with the true cardinalities.

$$
\begin{array}{lll}
n(S \bowtie R) & =n(S) \times \operatorname{fan}(\operatorname{S.own}[R])=1,000 \times \frac{50}{1,000}=50 & / * \text { forward traversal */ } \\
n(S \bowtie R) & =n(R) \times \operatorname{share}(\operatorname{S.own}[R])=2,000 \times \frac{50}{2,000}=50 & / * \text { backward traversal */ }
\end{array}
$$

Since the join is a partial participation join where students have only 50 trucks (including duplicates) among 1,000 vehicles (including cars, trucks, and duplicates), then the correct values of fan and share are $\frac{50}{1,000}$ and $\frac{50}{2,000}$, respectively; thus, the cardinality $n(S \bowtie R)$ becomes 50 .

In Eqs. (6) and (7), the parameter $n\left(C_{i} . A_{i}\left[D_{j}\right]\right)$ can be easily maintained in two ways: dynamically and statically. In dynamic maintenance, when inserting an object $o$ into $C_{i}$, we increment $n\left(C_{i} \cdot A_{i}\left[D_{j}\right]\right)$ by $k$ if the field $o . A_{i}$ contains $k$ Oids of $D_{j}$. Selecting only Oid's of $D_{j}$ is possible since each Oid includes the class identifier. When deleting an object $o$ from $C_{i}$, we decrement $n\left(C_{i} . A_{i}\left[D_{j}\right]\right)$ by $k$ if $o . A_{i}$ contains $k$ Oids of $D_{j}$. In the case of updates, the parameter can be maintained similarly: (1) If the old value in $o . A_{i}$ contains $k 1$ Oids of $D_{j}$, we decrement $n\left(C_{i} . A_{i}\left[D_{j}\right]\right)$ by $k 1$; (2) If the new value in $o . A_{i}$ contains $k 2$ Oids of $D_{j}$, we increment $n\left(C_{i} \cdot A_{i}\left[D_{j}\right]\right)$ by $k 2$. In static maintenance, we count $n\left(C_{i} \cdot A_{i}\left[D_{j}\right]\right)$ by accessing the objects of $C_{i}$ sequentially at a predefined interval or upon a user request. As each object is accessed, we add up the number of $D_{j}$ 's Oids contained in the attribute $A_{i}$. When this process finishes, the accumulated number of $D_{j}$ 's Oids becomes $n\left(C_{i} \cdot A_{i}\left[D_{j}\right]\right)$.

\subsection{Selectivity Estimation for Conditions}

Partial participation influences not only estimation of cardinalities for unconditional joins but also estimation of selectivities for conditions. In the previous section, we have not taken into account the selection conditions in the query imposed upon the classes to be joined. In this section, we discuss the problems of the conventional selectivity estimation techniques [19] when they are used for estimating the selectivities of conditions on partial participation classes. We then propose a new selectivity estimation technique that takes the effect of partial participation into account. The effect of multi-valued attributes is also taken into accounted in the proposed technique.

A nested predicate is a condition on a path expression of an arbitrary length [2]. If the length of the path expression is 1 , we call it a simple predicate [2]. Since object-oriented queries include nested predicates, a query optimizer has to estimate the selectivities of the nested predicates called path selectivities. We define the path selectivity as the ratio of the number of path instances that satisfy the predicate to the total number of path instances, i.e., the probability that a randomly selected path instance satisfies the predicate. 
As an example, let us consider the predicate $C_{i} \cdot A_{i}\left[D_{j}\right] \cdot A_{j}=$ "val" in Fig. 4 . Here, we assume that the domain of $A_{j}$ is a primitive class. In Fig. 4 , the grey-colored subregions (denoted by $D_{j}^{\prime}$ ) of $D_{j}$ denote the objects participating in the join with $C_{i}$, and the others non-participating objects. The selectivity of this nested predicate is the ratio of the number of path instances of the

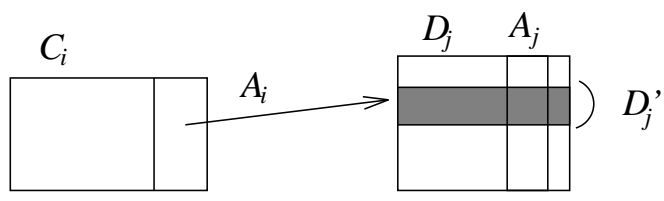

Fig. 4: Condition $C_{i} \cdot A_{i}\left[D_{j}\right] \cdot A_{j}=$ "val".

path $C_{i} \cdot A_{i}\left[D_{j}\right] \cdot A_{j}$ that satisfy $A_{j}=$ "val" to the total number of the path instances. Most of the conventional literature $[2,3,9,16,17]$ on object-oriented databases have no explicit estimation techniques for path selectivity. Kim et al. [11] use the technique proposed by Selinger et al.[19] for estimating the path selectivity as follows:

$$
\operatorname{sel}\left(C_{i} \cdot A_{i}\left[D_{j}\right] \cdot A_{j}=" v a l "\right)=\operatorname{sel}\left(D_{j} \cdot A_{j}=" v a l "\right)=\frac{1}{d v\left(D_{j} \cdot A_{j}\right)}
$$

However, if the codomain of the ending attribute (i.e., $A_{j}$ ) of the path expression (i.e., $D_{j}$ ) is a partial participation class, Eq. (10) produces an incorrect result. Specifically, since the path instances of the path expression $C_{i} \cdot A_{i}\left[D_{j}\right] \cdot A_{j}$ contain only the values of $A_{j}$ that belong to $D_{j}^{\prime}$ (denoted as $D_{j}^{\prime} \cdot A_{j}$ ), the probability that a path instance satisfies the predicate $C_{i} \cdot A_{i}\left[D_{j}\right] \cdot A_{j}=$ "val" should be estimated over the objects in $D_{j}^{\prime}$ instead of those in the entire $D_{j}$. The following example illustrates this point.

Example 5 In Fig. 1, we assume that the number of companies that produce cars is five among 500 companies: $\mathrm{dv}($ Car.manuf[Company] $)=5, \mathrm{n}($ Company $)=500$. The selectivity for Car.manuf[Company].name $=$ "Ford" is $1 / 5$, since there are only five companies that produce cars. However, the conventional method using Eq. (10) estimates the selectivity as $1 / 500$, which is equivalent to the selectivity of the simple predicate Company.name = "Ford".

We propose a new estimation technique for the path selectivity that considers the effects of partial participation. The key idea is to estimate the selectivity of the nested predicate $C_{i} \cdot A_{i}\left[D_{j}\right] \cdot A_{j}=$ "val" within $D_{j}^{\prime}$ instead of the entire $D_{j}$ as follows:

$$
\operatorname{sel}\left(C_{i} \cdot A_{i}\left[D_{j}\right] \cdot A_{j}=" v a l "\right)=\frac{1}{d v\left(C_{i} \cdot A_{i}\left[D_{j}\right] \cdot A_{j}\right)}=\frac{1}{d v\left(D_{j}^{\prime} \cdot A_{j}\right)}
$$

Here, $d v\left(C_{i} \cdot A_{i}\left[D_{j}\right] . A_{j}\right)$ is the number of distinct values in $D_{j} . A_{j}$ that are referred to by $C_{i} \cdot A_{i}$ (i.e., $\left.d v\left(D_{j}^{\prime} . A_{j}\right)\right)$. Hence, $1 / d v\left(C_{i} \cdot A_{i}\left[D_{j}\right] . A_{j}\right)$ is the probability that a path instance satisfies $A_{j}=$ "val". For example, in Example 5, Eq. (11) estimates the path selectivity for Car.manuf[Company].name $=$ "Ford" as follows:

$$
\operatorname{sel}(\text { Car.manuf.name }=\text { "Ford" })=\frac{1}{d v(\text { Car.manuf.name })}=\frac{1}{5} .
$$

As a special case, when the length of the path in a nested predicate is 1, Eq.(11) becomes the selectivity for the simple predicate, i.e., equivalent to Eq. (10).

Eq. (11) provides more accurate estimates than Eq. (10). For total participation (i.e., $D_{j}$ in Fig. 4 totally participates), the results of Eq. (10) and Eq. (11) are identical since $d v\left(C_{i} . A_{i}\left[D_{j}\right] \cdot A_{j}\right)$ is equal to $d v\left(D_{j} \cdot A_{j}\right)$. For partial participation, Eq. (11) estimates the selectivity greater than Eq. (10) by a factor of $\rho=\frac{d v\left(D_{j} \cdot A_{j}\right)}{d v\left(C_{i} \cdot A_{i}\left[D_{j}\right] \cdot A_{j}\right)}$ since $d v\left(D_{j} . A_{j}\right) \geq d v\left(C_{i} \cdot A_{i}\left[D_{j}\right] . A_{j}\right)$. As an example, in Example 5, since $\rho=\frac{d v(\text { Company.name })}{d v(\text { Car.manuf }[\text { Company }] . \text { name })}=\frac{500}{5}=100$, Eq. (11) estimates the selectivity one hundred times greater than Eq. (10). 
Compared with conventional ones, the proposed method requires more delicate statistics. The statistics used in Eq.(11) is $d v\left(C_{i} . A_{i}\left[D_{j}\right] . A_{j}\right)$; in contrast, the statistics used in Eq.(10) is $d v\left(D_{j} . A_{j}\right)$. The complexity of the former is higher than that of the latter. We propose two methods for computing these statistics efficiently: one is based on the nested index [2] and the other on the formula proposed by Yao [25].

(1) Method 1: Using a nested index

A nested index [2] establishes a direct connection between the first and the last objects in a path instance. It can be implemented using some variation of the B-tree data structure [2]. A non-leaf node consists of records, where each record is a triple (key length, key value, pointer). The pointer contains the physical address of the next-level index node. An index record in a leaf node consists of the record length, key length, key value, the list of Oids of the instances of the starting class that hold the key value in the indexed attribute(i.e., the ending attribute), and the number of elements in the list of Oids. A nested index $n$-indx defined on the path expression $C_{i} \cdot A_{i}\left[D_{j}\right] \cdot A_{j}$ has $d v\left(C_{i} \cdot A_{i}\left[D_{j}\right] \cdot A_{j}\right)$ key-values in the leaf nodes. Thus, with $n$-indx we can dynamically maintain the statistics as follows:

\section{Algorithm 1 (Statistics Maintenance)}

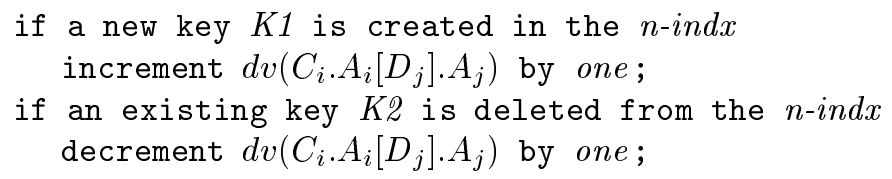

Other indices such as a path index [2] or an access support relation [8] can also be used instead of the nested index since these indices have more information than the nested index.

\section{(2) Method 2: Using Yao's formula}

If there is no nested indices on the path expression $C_{i} \cdot A_{i}\left[D_{j}\right] A_{j}$, we can compute the statistics by using Eq. (12) originally proposed by Yao [25].

Theorem 1 [25]: Given $\mathrm{n}$ records grouped into $\mathrm{m}$ blocks $(1 \leq \mathrm{m} \leq \mathrm{n})$, each containing $\mathrm{bf}=\mathrm{n} / \mathrm{m}$ records. If $\mathrm{k}$ records are randomly selected from the $\mathrm{n}$ records, the expected number of blocks hit (blocks with at least one record selected) is given by

$$
\begin{aligned}
& b(m, b f, k)=m \times\left(1-\frac{{ }_{n-b f} C_{k}}{{ }_{n} C_{k}}\right), \text { when } k \leq n-b f, \\
& b(m, b f, k)=m, \text { when } k>n-b f .
\end{aligned}
$$

Eq. (12) is originally intended to estimate the number of block accesses when a certain number of records are randomly selected. However, it can also be applied to estimating the number of distinct values in an attribute after selection operations [24]. We estimate $d v\left(C_{i} \cdot A_{i}\left[D_{j}\right] \cdot A_{j}\right)$ by using Eq. (12) as follows:

$$
d v\left(C_{i} \cdot A_{i}\left[D_{j}\right] \cdot A_{j}\right)=b\left(d v\left(D_{j} \cdot A_{j}\right), \frac{n\left(D_{j} \cdot A_{j}\right)}{d v\left(D_{j} \cdot A_{j}\right)}, d v\left(C_{i} \cdot A_{i}\left[D_{j}\right]\right)\right)
$$

Eq. (13) can be explained as follows. Objects in class $D_{j}$ are partitioned into $d v\left(D_{j} . A_{j}\right)$ groups, where each group has objects with the same value in the attribute $A_{j}$. These groups, called the logical groups [23], correspond to the number of blocks in Eq. (12). The parameter $\frac{n\left(D_{j} \cdot A_{j}\right)}{d v\left(D_{j} \cdot A_{j}\right)}$ represents the average number of objects in each group. It corresponds to the blocking factor in Eq. (12). The last parameter $d v\left(C_{i} \cdot A_{i}\left[D_{j}\right]\right)$ is the number of objects referred to by $C_{i} \cdot A_{j}$ and corresponds to the number of records to be selected in Eq. (12).

Since Eq. (12) involves an iterative form, the computation cost can be excessively high as $k$ becomes large. Approximation formulas for faster evaluation are presented in Whang et al. [23] and Diehr et al. [5]. 


\subsection{Cardinalities of Intermediate Results for $N$-way Joins}

In this section, we estimate intermediate results cardinalities for $n$-way joins (nested predicates involving $n$ classes) by using the cardinalities of unconditional joins and selectivity factors presented in Sections 4.2 and 4.3 , respectively.

A query involving $n$ classes may be joined in any order among $n$ ! permutations, just as $n$ relations may be joined in any order of $n$ ! permutations [12]. Let $\left\langle C_{1}, C_{2}, \ldots, C_{n}\right\rangle$ be a join sequence (permutation) of the set of $n$ classes in the query. We define the $k$-th intermediate result, denoted by $T_{k}$, in the join sequence $\left\langle C_{1}, C_{2}, \ldots, C_{n}\right\rangle$ as the result of joining the partial join sequence $\left\langle C_{1}, C_{2}, \ldots, C_{k}>\right.$. We also define the $k$-th intermediate result cardinality, denoted by $n\left(T_{k}\right)$, as the cardinality of $T_{k}$. We adopt the heuristic of avoiding Cartesian products as in [19]. Thus, among classes $C_{1}, \ldots, C_{k-1}$, class $C_{j}, 1 \leq j \leq k-1$, is directly connected to the next class $C_{k}$ [19]. Two classes are directly connected if one is the codomain and the other is the domain of an attribute. Then,

$$
\begin{aligned}
n\left(T_{k}\right) & =\left[n\left(T_{k-1}\right)\right] \times[\text { fan-out }(\text { or sharing level })] \times\left[\text { selectivity of } C_{k}\right] \\
& =n\left(T_{k-1}\right) \times \operatorname{fan}\left(C_{j} \cdot A_{j}\left[C_{k}\right]\right) \quad\left(\text { or share }\left(C_{k} \cdot A_{k}\left[C_{j}\right]\right)\right) \times \operatorname{sel}\left(C_{k}\right), k \geq 2 \\
n\left(T_{1}\right) & =n\left(C_{1}\right) \times \operatorname{sel}\left(C_{1}\right)
\end{aligned}
$$

where $\operatorname{sel}\left(C_{k}\right)$ is the product of selectivities of the selection conditions in the query that are applied to the class $C_{k}$.

Example 6 In Fig. 1, we estimate the intermediate results cardinalities for the nested predicate Student.own[Truck]manuf[Company].name $=$ "Ford" as follows. For convenience, we abbreviate the class name Student, Truck, and Company as S, R, and C, respectively. We assume the following statistics given in Table 1 .

Table 1: Statistics.

\begin{tabular}{||l|l|l||}
\hline S (Student) & $\mathrm{R}($ Truck $)$ & C (Company) \\
\hline \hline$n(S)=1,000$ & $n(R)=2,000$ & $n($ C $)=500$ \\
\hline$n($ S.own $)=1,500$ & $n($ R.manuf $)=2,000$ & $n($ C.name $)=500$ \\
\hline$n($ S.own $[R])=50$ & $n($ R.manu $[C])=2,000$ & $n($ C.name $[$ String $])=500$ \\
\hline$d v($ S.own $[R])=50$ & $d v($ R.manuf $[C])=5$ & $d v($ C.name $[$ String $])=500$ \\
\hline
\end{tabular}

1) $d v($ S.own $[R]$. manu $f[C]$. name $)$

- $d v(\operatorname{S.own}[R] . \operatorname{manuf}[C])$

$$
=b\left(d v(R . m a n u f[C]), \frac{n(R . m a n u f[C])}{d v(R . m a n u f[C])}, d v(S . o w n[R])\right)=b(5,400,50) \simeq 5
$$

- $d v(S . o w n[R] . m a n u f[C] . n a m e)$

$$
=b\left(d v(\text { C.name }), \frac{n(\text { C.name })}{d v(\text { C.name })}, d v(\text { S.own }[R] . \text { manu } f[C])\right)=b(500,1,5) \simeq 5
$$

2) Selectivities

- $\operatorname{sel}(S)=1 \quad \bullet \operatorname{sel}(R)=1$

- $\operatorname{sel}(C)=\operatorname{sel}($ S.own $[R] \cdot \operatorname{manu} f[C]$. name $=$ "Ford" $)=\frac{1}{d v(\text { S.own }[R] \cdot \operatorname{manuf}[C] \cdot \text { name })}=\frac{1}{5}$

3) Intermediate results cardinalities in the join sequence of $(S, R, C)$

- $n\left(T_{1}\right)=n(S) \times \operatorname{sel}(S)=1,000 \times 1=1,000$

- $n\left(T_{2}\right)=n\left(T_{1}\right) \times \operatorname{fan}(\operatorname{S.own}[R]) \times \operatorname{sel}(R)=1,000 \times \frac{n(S . \text { own }[R])}{n(S)} \times 1=50$

- $n\left(T_{3}\right)=n\left(T_{2}\right) \times \operatorname{fan}($ R.manu $[C]) \times \operatorname{sel}(C)=50 \times \frac{n(R . m a n u f[C])}{n(R)} \times \frac{1}{5}=10$

Actually, since there are 1,000 students and $\operatorname{sel}(S)=1, n\left(T_{1}\right)=1,000$. Since 1,000 students own 50 trucks $(n(\operatorname{S.own}[R])=50)$ and $\operatorname{sel}(R)=1, n\left(T_{2}\right)=50$. Since each truck has a manufacturer 
and only one fifth of them satisfy the condition "= Ford", $n\left(T_{3}\right)=10$. These are equivalent to our results.

In contrast, if we use conventional methods, the following are the cardinalities of intermediate results produced by using Eqs. (4) and (5). Note that conventional methods estimate $\operatorname{sel}(C)$ as $1 / 500$.

- $n\left(T_{1}\right)=n(S) \times \operatorname{sel}(S)=1,000 \times 1=1,000$

- $n\left(T_{2}\right)=n\left(T_{1}\right) \times \operatorname{fan}($ S.own $) \times \operatorname{sel}(R)=1,000 \times \frac{n(\text { S.own })}{n(S)} \times 1=1,500$

- $n\left(T_{3}\right)=n\left(T_{2}\right) \times \operatorname{fan}($ R.manuf $) \times \operatorname{sel}(C)=1,500 \times \frac{n(\text { R.manuf })}{n(R)} \times \frac{1}{500}=3$

These results show significent deviations from the true values for $n\left(T_{2}\right)$ and $n\left(T_{3}\right)$.

Based on these intermediate result cardinalities, we are able to estimate the cost of an evaluation plan for the nested predicate Student.own[Truck]manuf[Company].name = "Ford". For simplicity, we consider the evaluation plan of forward traversal pointer-based nested-loop join [11, 20]. Table 2 shows the comparison of the cost estimations. Here, the cost model proposed by Kim et al. [11] is used. Other join algorithms such as the sort-domain join or the hash join [11, 20] also

Table 2: Cost estimation for an evaluation plan.

\begin{tabular}{|c|c|c|}
\hline & proposed method & conventional methods \\
\hline $\operatorname{cost}\left(T_{1}\right)$ & $\begin{array}{c}\text { page(S) : number of page } \\
\text { accesses for Student }\end{array}$ & page(S) \\
\hline $\operatorname{cost}\left(T_{2}\right)$ & $\begin{array}{l}n\left(T_{1}\right) \times \operatorname{fan}(\text { S.own }[R]) \\
=1,000 \times \frac{50}{1,000} \\
=50 \text { page accesses }\end{array}$ & $\begin{array}{l}n\left(T_{1}\right) \times \operatorname{fan}(\text { S.own }) \\
=1,000 \times \frac{1,500}{1,000} \\
=1,500 \text { page accesses }\end{array}$ \\
\hline $\operatorname{cost}\left(T_{3}\right)$ & $\begin{array}{l}n\left(T_{2}\right) \times f a n(\text { R.manu } f[C]) \\
=50 \times \frac{2,000}{2,000} \\
=50 \text { page accesses }\end{array}$ & $\begin{array}{l}n\left(T_{2}\right) \times \text { fan }(\text { R.manuf }) \\
=1,500 \times \frac{2,000}{2,000} \\
=1,500 \text { page accesses }\end{array}$ \\
\hline evaluation cost & page $(\mathrm{S})+50+50$ page accesses & page $(\mathrm{S})+1,500+1,500$ page accesses \\
\hline
\end{tabular}

result in similar outcomes. The results show significant difference in cost estimation, which could lead the query optimizer to choose a solution that is far from the optimal one.

\section{CONCLUSIONS}

We have presented a new technique for estimating the intermediate results cardinalities of a query involving partial participation and multi-valued attributes. Although query optimization has been studied extensively in the literature, partial participation has not been considered seriously. The contributions of the paper can be summarized as follows.

First, we have argued that an object-oriented query often involves partial participation of classes in a relationship and compare the case with that for relational queries. Main causes of partial participation are codomain/domain substitutions and common domains, which are the characteristics of object-oriented databases.

Second, we have shown that conventional techniques may involve large estimation errors in estimating the intermediate results cardinalities when the query includes partial participation classes or multi-valued attributes. We have proposed new techniques for estimating the intermediate results cardinalities in which the effects of partial participation and multi-valued attributes are taken into accounted.

Finally, we have presented efficient methods for obtaining detailed statistics to be used for accommodating partial participation. Although these statistics are difficult to obtain in the relational databases, the proposed methods efficiently obtain those statistics by using inherent properties such as object identifiers and nested indices - of object-oriented databases. 
The most significant advantage of our technique is that the accuracy of the estimate for the cardinalities of unconditional joins and the selectivity factors is far enhanced with only a small overhead. Thus, the optimizer will be able to estimate the cost of an execution plan more accurately. As further studies, we are extending the selectivity estimation technique to consider range queries and are developing cost models for various other join algorithms.

Acknowledgements - We thank the reviewers for their careful comments on an earlier manuscript that helped enhance the clarity of this paper significantly. This work was partially supported by the Korean Ministry of Information and Communications through Korea Computer and Communications Co. as a part of the ODYSSEUS OODBMS project. It was also partially supported by the Korea Science and Engineering Foundation (KOSEF) through the Center for Artificial Intelligence Research. The last author was supported by the Brain Pool program of the Korean Foundation of Science and Technology Societies (KOFST). We would like to acknowledge the careful comments provided by Dr. K. H. Hong.

\section{REFERENCES}

[1] M. Atkinson, F. Bancilhon, D. DeWitt, K. Dittrich, D. Maier, and S. Zdonik. The object-oriented database system menifesto. In Proc. Int. Conf. on Deductive and Object-Oriented Databases, pp. 40-57, Kyoto, Japan (1989).

[2] E. Bertino and W. Kim. Indexing techniques for queries on nested objects. IEEE Trans. on Knowledge and Data Engineering, 1(2):196-214 (1989).

[3] J. A. Blakeley, W. J. McKenna, and G. G. Graefe. Experiences building the Open OODB query optimizer. In Proc. ACM SIGMOD Conference, pp. 287-296, Washington, DC (1993).

[4] S. Christodoulakis. Implications of certain assumptions in database performance evaluation. ACM Trans. on Database Systems, 9(2):163-186 (1984).

[5] G. Diehr and A. N. Saharia. Estimating block accesses in database organizations. IEEE Trans. on Knowledge and Data Engineering, 6(3):497-499 (1994).

[6] R. Elmasri and S. Navathe. Fundamentals of Database Systems. Benjamin/Cummings, 2nd edition (1994).

[7] Y.S. Ioannidis and S. Christodoulakis. On the propagation of errors in the size of join results. In Proc. ACM SIGMOD Conference, pp. 268-277 (1991).

[8] A. Kemper and G. Moerkotte. Access support in object bases. In Proc. ACM SIGMOD Conference, pp. 364-374, Atlantic City, New Jersey (1990).

[9] A. Kemper, G. Moerkotte, K. Peithner, and M. Steinbrunn. Optimizing disjunctive queries with expensive predicates. In Proc. ACM SIGMOD Conference, pp. 336-347, Minneapolis, Minnesota (1994).

[10] M. Kifer, W. Kim, and Y. Sagiv. Querying object-oriented databases. In Proc. ACM SIGMOD Conference, pp. 393-402, San Diego, CA. (1992).

[11] K. C. Kim, W. Kim, D. Woelk, and A. Dale. Acyclic query processing in object-oriented databases. In Proc. Intl. Conf. on Entity-Relationship Approach (1988).

[12] W. Kim. Introduction to Object-Oriented Databases. MIT Press (1990).

[13] W. Kim. Object-oriented database systems: Promises, reality, and futures. In W. Kim, editor, Modern Database Systems: The Object Model, Interoperability, and Beyond, pp. 255-280. Addision-Wesley (1995).

[14] W. Kim, K. C. Kim, and A. Dale. Indexing techniques for object-oriented databases. In W. Kim and F. Lochovsky, editors, Object-oriented Concepts, Applications, and Databases, pp. 371-394. Addision-Wesley (1989).

[15] K. H. Korth and A. Silberschatz. Database System Concepts. McGraw-Hill (1991).

[16] R. S. G. Lanzelotte and J-P. Cheiney. Adapting relational optimization technology to deductive and objectoriented declarative database languages. In Proc. Intl. Workshop on Database Programming Languages, pp. 322-335 (1991).

[17] D. Maier, S. Daniels, T. Keller, B. Vance, G. Graefe, and W. McKenna. Challenges for query processing in object-oriented databases. In J. C. Freytag, D. Maier, and G. Vossen, editors, Query Processing for Advanced Database Systems. Morgan Kaufmann (1994).

[18] M. V. Mannino, P. Chu, and T. Sagar. Statistical profile estimation in database systems. ACM Computing Surveys, 20(3):191-221 (1988).

[19] P. G. Selinger, M. M. Astrahan, D. D. Chamberlin, R. A. Lorie, and T. G. Price. Access path selection in a relational database management system. In Proc. ACM SIGMOD Conference, pp. 23-34, Boston, Mass. (1979).

[20] E. Shekita and M. Carey. A performance evaluation of pointer-based joins. In Proc. ACM SIGMOD Conference, pp. 364-374, Atlantic City, New Jersey (1990).

[21] K. Y. Whang. Constructing cost formulas for relational database query optimizers: A tutorial. In IEEE TENCON, pp. 132-141, Seoul, Korea (1987). 
[22] K. Y. Whang, B. T. Vander-Zanden, and H. M. Taylor. A linear time probabilistic counting algorithm for database applications. ACM Trans. on Database Systems, 15(2):208-229 (1990).

[23] K. Y. Whang, G. Wiederhold, and D. Sagalowicz. Estimating block accesses in database organizations - a closed noniterative formula. Comm. of the ACM, 26(11):940-944 (1983).

[24] K. Y. Whang, G. Wiederhold, and D. Sagalowicz. Separability - an approach to physical database design. IEEE Trans. on Computers, 33(3):209-222 (1984).

[25] S. B. Yao. Approximating block accesses in database organizations. Comm. of the ACM, 20(4):260-261 (1977). 